Brazilian Transportation

Planning Society
Journal of Transport Literature

Vol. 7, n. 2, pp. 171-191, Apr. 2013

Research Directory
JTL | RELIT

www.transport-literature.org ISSN 2238-1031

\title{
Seleção de uma aeronave para atividades de charter regional com utilização do método multicritério de borda modificado com utilização de mediana
}

[Commuter aircraft choice using a modified borda method using the median]

João Erick de Mattos Fernandes, Luiz Flávio Autran Monteiro Gomes, João Carlos Correia Baptista Soares de Mello, Silvio Figueiredo Gomes Júnior*

IBMEC, Brazil, IBMEC, Brazil, Universidade Federal Fluminense (UFF), Brazil,

Centro Universitário Estadual da Zona Oeste (UEZO), Brazil

Submitted 24 Jan 2012; received in revised form 25 May 2012; accepted 11 Jul 2012

\section{Resumo}

Este trabalho busca auxiliar na solução de um problema de seleção de uma aeronave enfrentado por uma empresa aérea que estuda o investimento na atividade de voo charter regional. A empresa em análise, pertencente a um grupo econômico cujo negócio principal é a logística, dispõe de oito alternativas a serem avaliadas sob onze diferentes critérios. A metodologia utilizada para a solução do problema é um novo método de apoio à decisão multicritério derivado do método de Borda, denominado Método de Borda com Utilização das Medianas. A utilização do método se justifica por sua característica de ser menos influenciado que o método original pelas alternativas irrelevantes e por sua facilidade de entendimento e baixa complexidade computacional. 0 método forneceu resultados consistentes, dando suporte confiável à decisão.

Palavras-Chave: aviação regional, método de borda modificado, análise multicritério, seleção de aeronave.

\begin{abstract}
This article tries to solve a problem of selecting an airship for an airline company whose core business is regional charter flights. The company belongs to an economic group whose main activity is logistics. The decision problem that the company faces has eight alternatives to be accessed according to eleven criteria. For approaching the problem a novel multicriteria decision aiding method is used. This new method is one variant of the classical Borda method denoted by Modified or Median-based Borda Method. Using this new method is justified by the fact that it is less influenced by the irrelevant alternatives as well as by its easy of understanding and its low computational complexity. The thus Modified Borda Method provided for consistent results and thus led to a reliable decision support.
\end{abstract}

Key words: regional aviation, modified borda method, multicriteria analysis, choice of an aircraft.

*Email: silviofgj@gmail.com.

\section{Recommended Citation}

Fernandes, J. E. M., Gomes, L. F. A. M., Mello, J. C. C. B. S. and Gomes Júnior, S. F. (2013) Seleção de uma aeronave para atividades de charter regional com utilização do método multicritério de borda modificado com utilização de mediana. Journal of Transport Literature, vol. 7, n. 2, pp. 171-191.

- JTL/RELIT is a fully electronic, peer-reviewed, open access, international journal focused on emerging transport markets and published by BPTS - Brazilian Transport Planning Society. Website www.transport-literature.org. ISSN 2238-1031. 


\section{Introdução}

A tomada de decisões é um processo natural e frequente para qualquer indivíduo ou instituição. Algumas decisões são simples e não implicam em grandes consequências, outras são mais complexas, exigindo um grau maior de expertise e uma metodologia coerente para a abordagem do problema, pois implicam em consequências mais profundas. Decisões complexas têm demandado abordagens estruturadas há muito tempo (Familytales, 2009). Este trabalho trata essencialmente de um problema de decisão, do tipo mais complexo, cuja importância demanda uma abordagem teoricamente estruturada.

O problema estudado diz respeito a uma empresa de transporte aéreo não regular. A empresa em estudo é uma empresa em formação, derivada de um grupo econômico cuja atividade principal é a logística. Fundado em 1969, o grupo é marcado por sua capacidade de diversificação, chegando a possuir, no ano de 2005, 12 empresas atuantes em diversos segmentos, variando de serviços de hotelaria a agronegócios.

No decorrer do processo de qualificação como operador aéreo, os gestores da empresa estudada vislumbraram a oportunidade de exploração do mercado de voos públicos não regulares, conhecidos como charter, dentro de rotas caracterizadas, tipicamente, como regionais. Nessa modalidade, são oferecidos ao público em geral assentos para destinos atendidos ou não por empresas aéreas regulares.

Dentre as análises necessárias para a formatação do novo produto a ser oferecido, os gestores identificaram como de vital importância para o sucesso do novo serviço a escolha correta da aeronave a ser utilizada nesse tipo de operação. A escolha de uma aeronave apresenta objetivos conflitantes, o que não possibilita a abordagem e decisão pela utilização de uma variável, apenas. Segundo Gomes (2007), a abordagem multicritério se faz necessária, dentre outros motivos, quando o número de objetivos a serem atendidos é igual ou maior que dois e estes entram em conflito entre si, como no caso analisado.

Assim, o objetivo deste trabalho é a utilização de um método de apoio à decisão multicritério, denominado Método de Borda Modificado com Utilização das Medianas como auxílio no processo de escolha de uma aeronave para a empresa alvo deste estudo. Gomes et al (2010) 
realizaram um estudo desse problema utilizando o Método NAIADE. O método proposto neste artigo tem a vantagem de exigir menos informação do decisor e ser menos sensível a posições extremas. Adicionalmente é de mais fácil compreensão e pouco exigente em termos computacionais.

\section{Métodos multicritério ordinais - Condorcet, Copeland e Borda}

Os primeiros métodos de Apoio à Decisão Multicritério datam formalmente da década de 1970. Contudo, ainda no século XVIII, durante a Revolução Francesa, surgiram os métodos ordinais de Borda e Condorcet, que podem ser considerados como precursores das escolas francesa e americana, elaboradas posteriormente (Soares de Mello et al, 2005a).

O Apoio à Decisão Multicritério consiste em um conjunto de métodos e técnicas para auxiliar ou apoiar a tomada de decisão, quando da presença de uma multiplicidade de critérios (Gomes, 2007). A forma de explicitar as estruturas de preferência do decisor varia de acordo com o método de análise multicritério escolhido. Os chamados métodos ordinais são considerados bastante intuitivos e pouco exigentes, tanto em termos computacionais, quanto em relação às informações necessárias por parte do decisor. Desse decisor não são exigidas mais do que as pré-ordens relativas a cada critério (Pomerol e Barba-Romero, 2000), ou seja, para o uso dos métodos ordinais, o decisor deve ordenar as alternativas de acordo com as suas preferências ou, eventualmente, usar uma ordenação natural como, por exemplo, a renda obtida.

Para a escolha do método ordinal é importante conhecer as suas características. Arrow (1950, 1951) sistematizou as condições necessárias para que um sistema não produzisse paradoxos e ao mesmo tempo fosse justo. Um método é considerado justo quando satisfaz a cinco axiomas:

- independência em relação às alternativas irrelevantes, ou seja, para todo par de alternativas x e y, as preferências entre x e y devem depender apenas de como as pessoas ordenam x em relação a y, e não de como ordenam as outras alternativas;

- ordem total (sem intransitividades e sem incomparabilidades); 
- unanimidade de Pareto, ou seja, se existe um par de alternativas, diga-se $\{A, B\}$, tal que todos os decisores preferem $\mathrm{A}$ à $\mathrm{B}$, então $\mathrm{A}$ deve ser posicionado, em termos de ordenação final, acima de B;

- transitividade, ou seja, se uma alternativa A é preferível à uma alternativa $\mathrm{B}$ e esta alternativa $\mathrm{B}$ é preferível em relação à alternativa $\mathrm{C}$, então a alternativa $\mathrm{A}$ é preferível à alternativa $\mathrm{C}$;

- universalidade.

No entanto, Arrow demonstrou, em seguida, a impossibilidade da existência de tal método. Assim, deve-se escolher o método que mais se adapte à situação analisada.

São de especial interesse no estudo a ser desenvolvido neste trabalho três dos axiomas de Arrow: os da independência em relação às alternativas irrelevantes, da transitividade e da universalidade. $\mathrm{O}$ primeiro afirma que a ordem de preferência entre duas alternativas não deve depender das suas preferências em relação a uma terceira alternativa. $O$ axioma da transitividade afirma que se uma alternativa é preferível a uma segunda, e esta a uma terceira, então a primeira deve ser preferível à terceira. Um exemplo um que não respeita o axioma da transitividade está relacionado aos resultados de jogos de futebol, e esse é o motivo da afirmação popular de que "futebol não tem lógica", pois, se um time A vence um time B e esse time $\mathrm{B}$ vence um time $\mathrm{C}$, nada garante que o time A vencerá o time C (o que deveria ocorrer caso o esporte respeitasse o axioma da transitividade). Já o axioma da universalidade exige que o método funcione, respeitando todos os outros axiomas, para qualquer conjunto de preferências dos decisores. Assim, um método que respeite os axiomas em alguns casos particulares, não respeita a universalidade.

$\mathrm{Na}$ literatura, os três métodos multicritério ordinais mais referenciados são os de Borda, Condorcet e Copeland, podendo aparecer variantes mais elaboradas dos métodos básicos (Levin e Nalebuff, 1995; Smith, 2006; Brandt, 2009). A grande vantagem da facilidade de uso e compreensão desses métodos é realçada por Kangas et al (2006) e Laukkanen et al (2004), que os aplicam a problemas de gestão florestal. Leskinen et al (2004) advertem para o perigo de se extrair mais informação do que se deve de resultados que combinam informações ordinal e cardinal. Valladares et al (2008) usam esses métodos num problema de agricultura. 
O método de Borda, considerado precursor da escola americana de multicritério (Gomes et al, 2004), que na essência é uma soma de pontos, tem a grande vantagem da simplicidade e, por isso, algumas de suas variantes são usadas em competições desportivas (Soares de Mello et al, 2005b; Kladroba, 2000). Rocha e Cavalcanti Netto (2002) o utilizaram como auxílio na avaliação de fornecedores de uma empresa petrolífera.

Para o uso do método de Borda, cada decisor deve ordenar as alternativas de acordo com as suas preferências. À alternativa preferida é atribuído um ponto, à segunda em ordem de preferência dois pontos e assim sucessivamente. Ao final, os pontos atribuídos pelos decisores a cada alternativa são somados e a alternativa que tiver obtido a menor pontuação será a escolhida (Dias et al, 1996). Todas as alternativas são ordenadas por ordem decrescente de pontuação (o que garante o respeito ao axioma da totalidade). Em esportes, variações do método de Borda são usadas com frequência, bastando para tal considerar cada competição como um decisor, e as suas preferências como a classificação final da competição. É normal fazer uma inversão do método, atribuindo maior número de pontos à alternativa mais preferida (concorrente vencedor da competição). Esta é uma modificação sem grande importância, mas outras, que geram algumas distorções, são também usadas. Destaque-se que um dos poucos exemplos de utilização do método de Borda original ocorre nas competições de iatismo, disputadas nos Jogos Olímpicos, onde os barcos recebem pontuação de acordo com a posição de chagada. Desta forma, o primeiro colocado ganha 1 ponto, o segundo 2 pontos e assim por diante, diferentemente de outros esportes em que o vencedor recebe a maior pontuação.

Apesar de sua simplicidade e amplo uso de suas variações, o método de Borda não respeita um dos axiomas de Arrow: a classificação final de duas alternativas não é independente em relação às suas classificações em relação a alternativas irrelevantes. Tal fato pode gerar situações indesejáveis, como numa votação em que o último votante sabe as preferências dos anteriores e altera as suas preferências, de modo a dar mais chances à sua alternativa preferida. Ou, estimular inversões anti-esportivas de posições numa competição para beneficiar um competidor como noticiado algumas vezes no Campeonato Mundial de Fórmula 1 (Soares de Mello et al, 2005b; Gomes Júnior e Soares De Mello, 2007). 
O método de Condorcet é considerado precursor da escola francesa de multicritério. Nele as alternativas são comparadas sempre duas a duas e constrói-se um grafo que expressa a relação entre elas. Nesse método também se exige que cada decisor ordene todas as alternativas de acordo com suas preferências. Todavia, ao invés de se atribuir uma pontuação a cada alternativa, o método estabelece relações de superação. Para estabelecer estas relações de superação, deve-se verificar, em cada par de alternativas, qual delas foi preferida pela maioria dos decisores. Nesse caso, diz-se que esta alternativa u é preferível em relação à outra, v. Podem ser traçados grafos representativos destas relações de preferência, em que o arco pertence ao grafo se, e só se, o número de decisores que preferiram u a v é maior ou igual ao dos que preferiram v a u. Esses resultados são análogos aos que seriam obtidos com o método ELECTRE I (Roy e Bouyssou, 1993), desde que não houvesse veto ou discordância, nem limiares de indiferença.

Através da representação da relação de preferência por um grafo, a determinação de alternativas dominantes e dominadas (quando existem), fica bastante facilitada. Quando existe uma e só uma alternativa dominante, ela é a escolhida. O método de Condorcet, considerado mais justo que o de Borda, tem a grande desvantagem de conduzir a situações de intransitividade, levando ao célebre "paradoxo de Condorcet". Esse ocorre quando uma alternativa $\mathrm{A}$ é preferível a outra $\mathrm{B}, \mathrm{B}$ é preferível à alternativa $\mathrm{C}$ e $\mathrm{C}$ é preferível a $\mathrm{A}$ (situação conhecida como "Tripleta de Condorcet”, ilustrada na Figura 1).

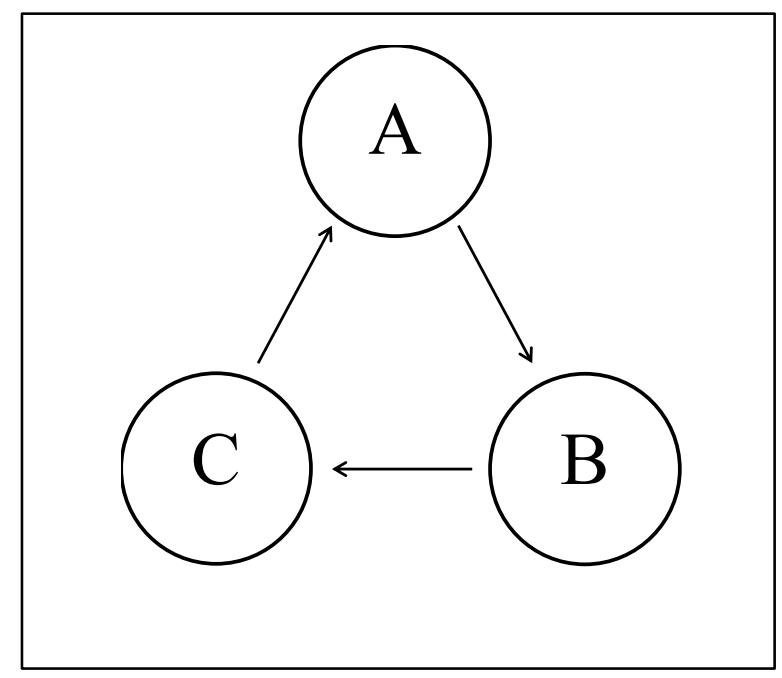

Figura 1 - Tripleta de Condorcet ${ }^{1}$

\footnotetext{
${ }^{1}$ Fonte: elaboração própria.
} 
Isto significa que o método de Condorcet nem sempre induz uma pré-ordem no conjunto das alternativas. Se o objetivo for realizar uma escolha, mesmo com intransitividades, o método de Condorcet tem uma vantagem: obriga a intervenções interativas com o decisor, evitando o paradigma do ótimo. Esse paradigma é criticado em Clímaco (2003). No entanto, existem aplicações em que não ocorrem ciclos de intransitividade, como por exemplo, em votações políticas em que os eleitores tenham uma coerência coletiva, ordenando as alternativas da mais à esquerda para a mais à direita, ou o contrário. Em situações análogas a esta, o método de Condorcet deve ser preferido ao método de Borda. Entretanto, vale lembrar que um método multidecisor deve respeitar o axioma da universalidade, e isto não acontece com o de Condorcet.

A escolha entre os métodos de Borda e Condorcet é uma escolha entre permitir situações de possível manipulação de resultados ou de dificuldades para obter um resultado completo.

O método de Condorcet utiliza uma matriz de adjacência para a ordenação das alternativas. Um exemplo desta matriz pode ser visto na Figura 2, que apresenta a matriz de adjacência para ordenação dos aeroportos brasileiros retirada do trabalho de Pinheiro e Soares de Mello (2005).

\begin{tabular}{|c|c|c|c|c|c|c|c|c|c|c|c|c|c|c|c|c|c|}
\hline Aeroporto & Sigla & $\mathrm{CGH}$ & GRU & BSB & GIG & SSA & REC & SDU & CWB & POA & FOR & PLU & BEL & MAO & FLN & GYN & $\mathrm{VCP}$ \\
\hline Congonhas & $\mathrm{CGH}$ & 0 & 1 & 1 & 1 & 1 & 1 & 1 & 1 & 1 & 1 & 1 & 1 & 1 & 1 & 1 & 1 \\
\hline Guarulhos & GRU & 0 & 0 & 1 & 1 & 1 & 1 & 1 & 1 & 1 & 1 & 1 & 1 & 1 & 1 & 1 & 1 \\
\hline Brasília & BSB & 0 & 0 & 0 & 1 & 1 & 1 & 1 & 1 & 1 & 1 & 1 & 1 & 1 & 1 & 1 & 1 \\
\hline Galeão & GIG & 0 & 0 & 0 & 0 & 1 & 1 & 1 & 1 & 1 & 1 & 1 & 1 & 1 & 1 & 1 & 1 \\
\hline Salvador & SSA & 0 & 0 & 0 & 0 & 0 & 1 & 1 & 1 & 1 & 1 & 1 & 1 & 1 & 1 & 1 & 1 \\
\hline Recife & REC & 0 & 0 & 0 & 0 & 0 & 0 & 1 & 1 & 1 & 1 & 1 & 1 & 1 & 1 & 1 & 1 \\
\hline Santos-Dumont & SDU & 0 & 0 & 0 & 0 & 0 & 0 & 0 & 1 & 1 & 1 & 1 & 1 & 1 & 1 & 1 & 1 \\
\hline Curitiba & CWB & 0 & 0 & 0 & 0 & 0 & 0 & 0 & 0 & 1 & 1 & 1 & 1 & 1 & 1 & 1 & 1 \\
\hline Porto Alegre & POA & 0 & 0 & 0 & 0 & 0 & 0 & 0 & 0 & 0 & 1 & 1 & 1 & 1 & 1 & 1 & 1 \\
\hline Fortaleza & FOR & 0 & 0 & 0 & 0 & 0 & 0 & 0 & 0 & 0 & 0 & 1 & 1 & 1 & 1 & 1 & 1 \\
\hline Pampulha & PLU & 0 & 0 & 0 & 0 & 0 & 0 & 0 & 0 & 0 & 0 & 0 & 1 & 1 & 1 & 1 & 1 \\
\hline Belém & BEL & 0 & 0 & 0 & 0 & 0 & 0 & 0 & 0 & 0 & 0 & 0 & 0 & 1 & 1 & 1 & 1 \\
\hline Manaus & MAO & 0 & 0 & 0 & 0 & 0 & 0 & 0 & 0 & 0 & 0 & 0 & 0 & 0 & 1 & 1 & 1 \\
\hline Florianópolis & FLN & 0 & 0 & 0 & 0 & 0 & 0 & 0 & 0 & 0 & 0 & 0 & 0 & 0 & 0 & 1 & 1 \\
\hline Goiânia & GYN & 0 & 0 & 0 & 0 & 0 & 0 & 0 & 0 & 0 & 0 & 0 & 0 & 0 & 0 & 0 & 1 \\
\hline Campinas & $\mathrm{VCP}$ & 0 & 0 & 0 & 0 & 0 & 0 & 0 & 0 & 0 & 0 & 0 & 0 & 0 & 0 & 0 & 0 \\
\hline
\end{tabular}

Figura 2 - Matriz de Adjacência dos Aeroportos Brasileiros ${ }^{2}$

\footnotetext{
${ }^{2}$ Fonte: Pinheiro e Soares de Mello (2005).
} 
Quando existem ciclos de intransitividade no método de Condorcet, onde não se consegue estabelecer uma ordenação das alternativas, o método de Copeland permite fazer a ordenação e mantém a ordenação das alternativas que não pertencem a nenhum ciclo de intransitividade. O método de Copeland usa a mesma matriz de adjacência que representa o grafo do método de Condorcet. A partir dela, calcula-se a soma das vitórias menos as derrotas, em uma votação por maioria simples. As alternativas são então ordenadas pelo resultado decrescente dessa soma. O método de Copeland alia a vantagem de sempre fornecer uma ordenação total (ao contrário do de Condorcet), ao fato de dar o mesmo resultado de Condorcet, quando esse não apresenta nenhum ciclo de intransitividade. Apesar de computacionalmente mais exigente que o de Borda, quando há necessidade de se estabelecer uma relação de pré-ordem, ou ordem latus sensu, esse método fornece sempre uma resposta (o que não acontece com o método de Condorcet) e, apesar de não eliminar, reduz bastante a influência de alternativas irrelevantes. O método de Copeland pode ser considerado um compromisso entre as filosofias opostas de Borda e Condorcet, reunindo, dentro do possível, as vantagens dos dois.

No entanto, os métodos de Condorcet e Copeland são bastante complexos para o entendimento dos gestores da empresa. A construção da matriz de Condorcet, que serve de base para os 2 métodos é, além disso, muito trabalhosa. Por esta razão, propõe-se neste trabalho um método alternativo ao Método de Borda, denominado Método de Borda Modificado com Utilização das Medianas, para estabelecer a ordenação das alternativas do problema. Nesse método, ao invés da ordenação das alternativas ser realizada utilizando a pontuação total como normalmente se faz no método de Borda, os autores propõem a utilização da mediana de cada alternativa para se realizar esta ordenação. Esta técnica deixa o método bastante simples, entretanto busca reduzir a influência das alternativas irrelevantes na ordenação final.

O método consiste então em estabelecer a ordenação das alternativas segundo cada um dos critérios utilizados no estudo. Após as ordenações em cada critério separadamente, deve-se verificar qual a posição de cada uma das alternativas nestas ordenações, colocando-as em ordem crescente de posição. O valor da mediana utilizada no método para a ordenação final das alternativas é o valor da mediana de cada alternativa segundo as ordenações individuais. Esse método, pelo fato das medianas não serem influenciadas pelos valores extremos, é menos dependente das alternativas irrelevantes que o método de Borda. Entretanto, é mais 
dependente dessas alternativas que o método de Copeland, mas sua utilização é compensada pela sua simplicidade.

Logo, por ser menos influenciado pelas alternativas irrelevante que o método de Borda e mais simples de ser utilizado que o método de Copeland, o Método de Borda Modificado com utilização das Medianas foi o método escolhido para auxiliar os decisores na escolha da aeronave para a empresa em questão.

\section{Definição do Problema}

A empresa em estudo surgiu em 1983 com o objetivo de produzir aeronaves ultraleves, para a prática do aerodesporto. Desde sua fundação, esta empresa teve sua localização no aeródromo de Maricá, no Estado do Rio de Janeiro. Após ser adquirida por um grupo altamente diversificado, em 1991, a organização sofreu um desinvestimento, cessando posteriormente suas atividades. A empresa permaneceu inativa até o ano de 2007, mantendo seu patrimônio por meio da sublocação de suas dependências. No final desse mesmo ano, foram iniciados estudos para a reativação da empresa, visando o aproveitamento das edificações disponíveis para a instalação de uma empresa do ramo aeronáutico, tendo-se decidido por uma empresa de taxi aéreo. Os regulamentos para homologação de empresa de taxi aéreo possibilitam a exploração de voos de passageiros sob demanda, modalidade conhecida como charter. Aproveitando-se disto, os gestores da empresa propuseram uma avaliação, com o objetivo de formular um planejamento econômico para a adoção desse tipo de operação.

O problema crítico encontrado nesta avaliação foi o de escolha de uma aeronave, já que representava a principal parte do investimento necessário, superando até o investimento inicial calculado para as operações. Tal montante poderia ser vital para uma empresa com a escala da estudada (Bercovitz e Mitchell, 2007).

Além disto, o processo de escolha também se mostrava complexo no primeiro momento, dado o número de critérios e suas variações de métrica, e também do universo de alternativas a serem escolhidas. 
Justifica-se, portanto, pela importância para a empresa e complexidade do problema de decisão, a adoção de uma ferramenta de apoio multicritério à decisão, que possibilite aos gestores uma tomada de decisão fundamentada.

Como pertence a um grupo econômico, a decisão fica a cargo do presidente desse grupo. As informações repassadas ao analista de decisão são provenientes, sobretudo, de pesquisas internas da empresa, ficando ainda por conta do diretor de operações a avaliação do valor das variáveis qualitativas necessárias ao processo e que foram tratadas por meio da lógica nebulosa, ou fuzzy (Zadeh, 1975), mas que não está no escopo deste trabalho. Coube a esses dois atores, auxiliados pelo analista de decisão, fazer a definição do conjunto de alternativas e da família de critérios. Para auxiliar na resolução do problema, um método multicritério de apoio a decisão, com qualidades mais alinhadas àquelas que demanda o problema, foi escolhido.

Como mencionado na seção 2, o método multicritério escolhido é um método alternativo ao Método de Borda denominado Método de Borda Modificado com Utilização das Medianas, pois entende-se que os métodos de Condorcet e Copeland são bastante complexos para o entendimento dos gestores da empresa. Além disso, o método proposto busca ser mais independente em relação às alternativas irrelevantes, minimizando as distorções do Método de Borda original.

Um outro método que se adapta bem a situações desse tipo é o VIP Analysis (Dias e Clímaco, 2000). No entanto tem as desvantagens de exigir a prévia construção de funções de valor precisas e de exigir independência preferencial entre os critérios, o que levou à sua não utilização neste trabalho. A utilização de outros métodos multicritério para resolver outros problemas ligados ao transporte aéreo podem ser vistos nos trabalhos de Wang e Chang (2007),Wang (2008), Yoo e Choi (2006), Chang e Yeh (2001, 2002), Soares de Mello et al (2003), Teodorović (1985), Rahman e Gomes (2008), Soares de Mello et al (2005a) e Silveira et al (2008). 


\section{Metodologia}

A ANAC - Agência Nacional de Aviação Civil é a autoridade Brasileira responsável pela regulação do transporte aéreo. Constituída pela lei 11.182 (Brasil, 2005), a ANAC regula o setor amparada pelo Código Brasileiro de Aeronáutica (Brasil, 1986) e regulamentos específicos, denominados Regulamentos Brasileiros de Homologação Aeronáutica - RBHA.

Segundo os RBHA 119 (Brasil, 2003a) e 135 (Brasil, 2003b), as aeronaves possíveis para a operação de charter regional são aquelas de capacidade igual ou inferior a 30 passageiros, o que já limita significativamente as alternativas viáveis.

Ainda segundo esses regulamentos, algumas vantagens podem ser obtidas por empresas que optam pela utilização de aeronaves com capacidade igual ou inferior a 19 passageiros. A principal vantagem pode ser encontrada no RBHA 135.3(b) (Brasil, 2003b), onde a empresa fica dispensada de treinamento mais detalhado da tripulação. Outra vantagem significativa pode ser encontrada em RBHA 135.107 (Brasil, 2003b), que dispensa a empresa da necessidade de um comissário qualificado a bordo de seus vôos, o que reduz significativamente os custos operacionais, de treinamento e de confecção e manutenção dos documentos necessários à operação. Com esse novo corte, reduz-se ainda mais o universo de escolhas possíveis. Segundo os gestores da empresa, as alternativas a serem avaliadas são: Cessna Caravan, Fairchild Metro, Beechcraft 1900, Embraer EMB-110 Bandeirante, LET 410, DeHavilland DHC 6, Dornier 228 e CASA 212. Segue uma breve descrição de cada uma destas aeronaves, segundo o sítio Airliners.net (2009):

- Cessna Caravan. Originário dos Estados Unidos da América, esta aeronave foi concebida no início da década de 80, tendo realizado seu primeiro voo em 1982 e entrado em operação três anos mais tarde. A aeronave é um monomotor turbo-hélice com capacidade para até doze passageiros. Sua produção ainda é ativa e seu maior operador é a empresa americana FedEx.

- Fairchild Metro. Também nasceu nos Estados Unidos, seu projeto data do início dos anos 60, tendo entrado em operação no ano de 1973. Sua configuração é bimotora turbo-hélice, com capacidade para dezenove passageiros. 
- Beechcraft 1900. Concebido nos Estados Unidos em 1979, teve sua entrada em serviço em 1984. É uma aeronave bimotora turbo-hélice, com capacidade para dezenove passageiros.

- Embraer EMB-110 Bandeirante. Foi idealizado no Brasil antes mesmo da fábrica que o produziu. O Bandeirante ou "Bandit", como ficou conhecido no mercado internacional, entrou em operação em 1973, sendo uma aeronave bimotora, turbohélice, para dezoito passageiros.

- LET 410. Projetado na República Tcheca em 1966, esta aeronave voou pela primeira vez em 1969. Sua produção ainda continua ativa. É uma aeronave bimotora, turbohélice, para dezenove passageiros.

- DeHavilland DHC-6. A aeronave comercial mais bem sucedida do Canadá, o DHC-6 entrou em serviço em 1966. É uma aeronave bimotora turbo-hélice, com capacidade para até vinte passageiros. Seu projeto foi comprado pela empresa Viking Air, também no Canadá, que deu continuidade em sua produção.

- Dornier 228. Origina-se da Alemanha, tendo entrado em serviço em 1982. É uma aeronave bimotora, turbo-hélice, com capacidade máxima de dezenove passageiros.

- CASA 212. É um projeto Espanhol, cuja primeira aeronave comercial entrou em serviço em 1975. O Aviocar, como é conhecido, é uma aeronave bimotora, turbohélice, com capacidade máxima de 26 passageiros (em configuração transporte militar). Sua produção foi continuada na Indonésia pela empresa ITPN (Indonesian Aerospace).

No que diz respeito aos critérios, Gomes (2007), afirma que uma das possibilidades de trabalhar com a definição dos critérios é criando uma hierarquia em forma de árvore, ficando assim bastante similar ao encontrado no método AHP (SAATY, 2005). Nos níveis mais superiores ficam os critérios abrangentes, que são subdivididos em outros critérios mais detalhados, até que se forme uma família de critérios suficientemente específicos para que possam ser efetivamente avaliados. A Figura 3 retrata esta subdivisão, já como um produto final da definição por parte dos gestores e depuração por parte do analista de decisão. 


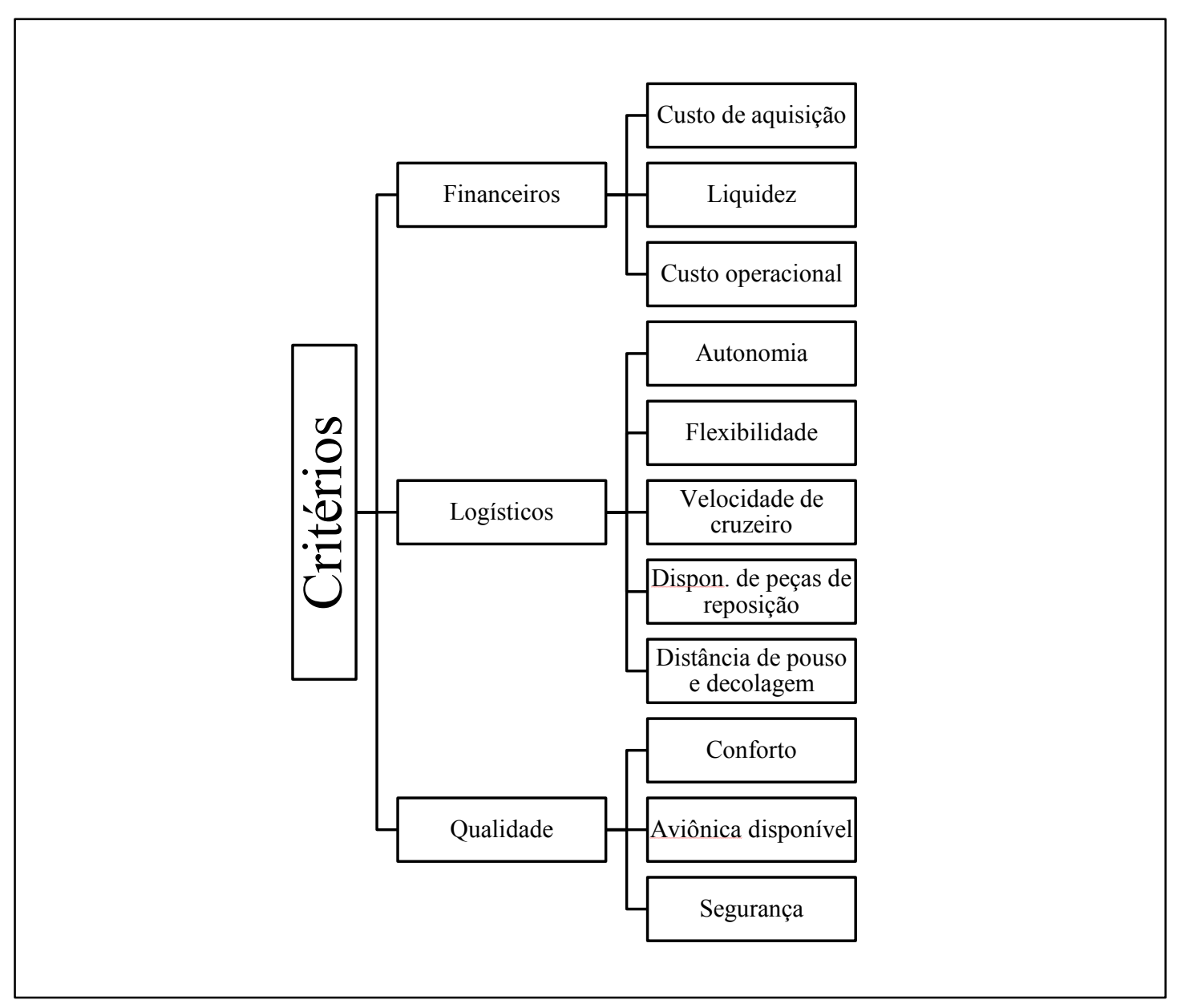

Figura 3 - Subdivisão em níveis dos Critérios ${ }^{3}$

No primeiro nível, os critérios são divididos em três grupos. No grupo financeiro, figuram os critérios que implicam em desembolsos diretos, ou que produzem algum impacto na administração financeira ou econômica da instituição. No grupo de critérios ligados à logística estão listados os critérios mais significativos para a operação das aeronaves e as suas limitações. Já no grupo ligado à qualidade, os critérios refletem, de maneira direta ou indireta o nível de satisfação relativo, por parte dos usuários da alternativa.

No segundo nível, estão presentes os critérios que serão efetivamente avaliados. Para um melhor entendimento da importância de cada critério dentro do modelo, segue uma breve descrição de cada um deles:

\footnotetext{
${ }^{3}$ Fonte: elaboração própria.
} 
- Custo de Aquisição. O custo de aquisição é o valor pago pela aquisição, transporte e legalização da aeronave pronta para a operação. Figuram também nesse critério quaisquer outros custos necessários para que a aeronave esteja aeronavegável em território brasileiro. É medido em termos monetários.

- Liquidez. A Liquidez é um critério que ajuda a qualificar a flexibilidade financeira em relação às aeronaves como ativo. Em outras palavras, a liquidez define o quão facilmente o ativo pode ser convertido em valores monetários. Nesse critério, são observados o tempo e o valor usual de revenda das aeronaves. Trata-se de um critério qualitativo, portanto, não possui uma unidade de medida.

- Custo operacional. Os custos operacionais traduzem os custos diretos de operação da aeronave, como combustível e desgaste físico de peças e equipamentos. Os valores para esse critério são medidos em valores monetários por milha por passageiro.

- Autonomia. Primeiro critério do grupo logístico, a autonomia é distância que a aeronave pode percorrer sem reabastecimento. Dada a variação do preço do combustível em relação ao seu custo de transporte, a autonomia pode ser uma grande vantagem na administração dos custos com abastecimento, além de dar mais flexibilidade na operação, com a possibilidade de exploração de rotas mais longas. A autonomia é medida em termos de distância, mais especificamente milhas náuticas.

- Flexibilidade. Trata-se de um critério de natureza qualitativa, que leva em conta a diversidade de terrenos em que a aeronave opera, bem como o seu número de classificação, segundo o método ACN-PCN, desenvolvido pela Organização da Aviação Civil Internacional e publicado em seu Anexo 14 (ICAO, 2004). Cada aeronave possui um ACN (Número de Classificação de Aeronave) e quanto menor esse número, menor é a resistência necessária do pavimento das pistas de pouso, e, portanto, maior é o número de aeroportos em que se pode operar. PCN representa o Número de Classificação de Pavimento.

- Velocidade de Cruzeiro. A velocidade de cruzeiro, além de ser um critério importante na qualidade, já que a mais significativa característica do transporte aéreo é a velocidade, carrega também uma grande importância em termos logísticos, já que aeronaves mais rápidas são capazes de cumprir mais etapas em relação às aeronaves mais lentas. Seu valor é medido em nós (milhas náuticas por hora). 
- Disponibilidade de Peças de Reposição. Esse critério é um esforço para refletir a proximidade de fornecedores de peças de reposição e o tempo necessário para adquirir esse material quando necessário. Por ser de mensuração difícil, o critério reflete termos relativos, sendo, portanto, de natureza qualitativa.

- Distância de pouso e decolagem. Uma das limitações impostas à operação de rotas para certas localidades é o tamanho da pista de pouso disponível. A operação pode tornar-se inviável se a pista for demasiadamente curta. A distância de pouso de decolagem, portanto, figura como um critério relevante, já que a alternativa mais desejável é aquela que possibilita a operação em pistas de tamanho reduzido. A distância será medida em metros.

- Conforto. Para a mensuração de conforto para os passageiros na cabine, optou-se pelo volume da cabine. O volume traduz o quão amplo é o espaço disponível para os passageiros e é medido em metros cúbicos.

- Aviônica disponível. Nesse critério fica definida a possibilidade de incorporação de novas tecnologias à alternativa. É um critério de natureza qualitativa, não possuindo unidades de medida.

- Segurança. Utilizou-se uma análise qualitativa da percepção de segurança, tanto em termos técnicos quanto da parte do cliente, em relação a cada uma das alternativas.

\section{Aplicação e Resultados}

A Tabela 1 apresenta os dados do problema, trazendo os valores apurados para cada alternativa em cada critério.

Como descrito na Seção 2, o método utilizado neste estudo é o Método de Borda Modificado com Utilização das Medianas. Esse método é uma variação do Método de Borda original e faz com que as alternativas sejam ordenadas por meio de suas medianas, em ordem crescente ao invés da soma das pontuações de cada alternativa como no método original. Essa variação permite que o método seja menos influenciado pelas alternativas irrelevantes e pelas posições extremas, minimizando as distorções do Método de Borda. Além disso, torna-o de mais fácil compreensão para os gestores da empresa em relação aos métodos de Condorcet e Copeland. 
Tabela 1 -Valores para critérios ${ }^{4}$

\begin{tabular}{|c|c|c|c|c|}
\hline & Cessna 208 & DHC-6 & LET 410 & Metro \\
\hline C. $\mathrm{Aq}$ & $\mu=1000 \mathrm{~K} \sigma=150 \mathrm{~K}$ & $\mu=1900 \mathrm{~K} \sigma=400 \mathrm{~K}$ & $\mu=700 \mathrm{~K} \sigma=200 \mathrm{~K}$ & $\mu=1000 \mathrm{~K} \sigma=200 \mathrm{~K}$ \\
\hline Liquidez & Perfeito & Moderado & Muito Bom & Razoavel Ruim \\
\hline C. Op. & 0,2475 & 0,3116 & 0,2574 & 0,2911 \\
\hline Autonomia & 917 & 775 & 755 & 595 \\
\hline Flex & Muito Bom & Bom & Bom & Razoavel Bom \\
\hline Vel. Cruz. & 184 & 182 & 208 & 242 \\
\hline Disp. Peças & Muito Bom & Moderado & Bom & Razoavel Ruim \\
\hline Dist. Pouso & 738 & 366 & 935 & 625 \\
\hline Conforto & 9,60 & 10,87 & 17,90 & 17,92 \\
\hline Aviônica & Muito Bom & Bom & Muito Bom & Razoavel Ruim \\
\hline \multirow[t]{2}{*}{ Segurança } & Bom & Muito Bom & Muito Bom & Bom \\
\hline & Beech 1900 & EMB 110 & Dornier 228 & CASA 212 \\
\hline C. $\mathrm{Aq}$ & $\mu=2000 \mathrm{~K} \sigma=250 \mathrm{~K}$ & $\mu=1000 \mathrm{~K} \sigma=200 \mathrm{~K}$ & $\mu=1250 \mathrm{~K} \sigma=250 \mathrm{~K}$ & $\mu=900 \mathrm{~K} \sigma=200 \mathrm{~K}$ \\
\hline Liquidez & Moderado & Bom & Razoavel Ruim & Bom \\
\hline C. Op. & 0,2995 & 0,3011 & 0,2889 & 0,2953 \\
\hline Autonomia & 1100 & 980 & 560 & 820 \\
\hline Flex & Bom & Bom & Razoave Bom & Muito Bom \\
\hline Vel. Cruz. & 248 & 190 & 271 & 180 \\
\hline Disp. Peças & Perfeito & Perfeito & Razoavel Ruim & Razoavel Ruim \\
\hline Dist. Pouso & 1050 & 980 & 450 & 675 \\
\hline Conforto & 16,20 & 12,94 & 14,63 & 24,35 \\
\hline Aviônica & Muito Bom & Bom & Razoavel Ruim & Muito Bom \\
\hline Segurança & Muito Bom & Muito Bom & Bom & Muito Bom \\
\hline
\end{tabular}

A Tabela 2 apresenta as pontuações atribuídas a cada uma das alternativas (aeronaves) segundo cada um dos critérios apresentados na Figura 3 para sua ordenação. Esses critérios estão numerados na tabela pela seguinte ordem: 1 - Custos de aquisição; 2 - Liquidez; 3 Custo operacional; 4 - Autonomia; 5 - Flexibilidade; 6 - Velocidade de cruzeiro; 7 Disponibilidade de peças de reposição; 8 - Distância de pouso e decolagem; 9 - Conforto; 10 - Aviônica disponível e 11 - Segurança.

\footnotetext{
${ }^{4}$ Fonte: elaboração própria.
} 
Tabela 2 - Ordenação das alternativas segundo cada critério ${ }^{5}$

\begin{tabular}{|c|c|c|c|c|c|c|c|c|c|c|c|}
\hline \multirow{2}{*}{ Alternativas } & \multicolumn{11}{|c|}{ Critérios } \\
\hline & 1 & 2 & 3 & 4 & 5 & 6 & 7 & 8 & 9 & 10 & 11 \\
\hline Cessna Caravan & 4 & 1 & 1 & 3 & 1,5 & 6 & 3 & 5 & 8 & 2,5 & 7 \\
\hline Fairchild Metro & 7 & 5,5 & 8 & 5 & 4,5 & 7 & 5 & 1 & 7 & 5,5 & 3 \\
\hline Beechcraft 1900 & 1 & 2 & 2 & 6 & 4,5 & 4 & 4 & 6 & 3 & 2,5 & 3 \\
\hline $\begin{array}{l}\text { Embraer EMB- } \\
110 \text { Bandeirante }\end{array}$ & 4 & 7,5 & 4 & 7 & 7,5 & 3 & 7 & 3 & 2 & 7,5 & 7 \\
\hline LET 410 & 8 & 5,5 & 6 & 1 & 4,5 & 2 & 1,5 & 8 & 4 & 2,5 & 3 \\
\hline $\begin{array}{c}\text { DeHavilland } \\
\text { DHC } 6\end{array}$ & 4 & 3,5 & 7 & 2 & 4,5 & 5 & 1,5 & 7 & 6 & 6,5 & 3 \\
\hline Dornier 228 & 6 & 7,5 & 3 & 8 & 7,5 & 1 & 7 & 2 & 5 & 7,5 & 7 \\
\hline CASA 212 & 2 & 3,5 & 5 & 4 & 1,5 & 8 & 7 & 4 & 1 & 2,5 & 3 \\
\hline
\end{tabular}

A etapa seguinte consiste na aplicação do método escolhido. Foi identificada a mediana da pontuação dos critérios para cada uma das alternativas. Esse valor encontrado foi ordenado em ordem crescente e, assim, ordenadas também as alternativas do problema. Para comparação dos resultados obtidos, as alternativas também foram ordenadas segundo o método de Borda original. Os resultados encontrados por esses dois métodos encontram-se apresentados da Tabela 3.

Tabela 3 - Ordenação segundo método de Borda modificado e Borda original ${ }^{6}$

\begin{tabular}{ccccc}
\hline Alternativas & $\begin{array}{c}\text { Posição } \\
\text { Mediana }\end{array}$ & $\begin{array}{c}\text { Ordenação } \\
\text { Mediana }\end{array}$ & $\begin{array}{c}\text { Pontuação } \\
\text { Borda }\end{array}$ & $\begin{array}{c}\text { Ordenação } \\
\text { Borda }\end{array}$ \\
\hline Cessna Caravan & 3 & 1 & 42 & 3 \\
Fairchild Metro & 5,5 & 6 & 58,5 & 6 \\
Beechcraft 1900 & 3 & 1 & 38 & 1 \\
Embraer EMB-110 & 7 & 7 & 59,5 & 7 \\
Bandeirante & 4 & 4 & 46 & 4 \\
LET 410 & 4,5 & 5 & 50 & 5 \\
DeHavilland DHC 6 & 7 & 7 & 61,5 & 8 \\
Dornier 228 & 3,5 & 3 & 41,5 & 2 \\
CASA 212 & & & & \\
\hline
\end{tabular}

\footnotetext{
${ }^{5}$ Fonte: elaboração própria.

${ }^{6}$ Idem.
} 
Pelos resultados apresentados na Tabela 3, pode-se verificar que a aeronave Beechcraft é a melhor alternativa nos dois métodos. No entanto, esta aeronave empata com o Cessna Caravan no método de Borda modificado. As demais alternativas permanecem inalteradas na ordenação. Vale ressaltar que a aeronave Casa 212 só muda para a $3^{\mathrm{a}}$ colocação no método de Borda modificado devido ao empate ocorrido na $1^{\mathrm{a}}$ colocação. O empate ocorrido no método de Borda modificado já era esperado uma vez que, como descrito na Seção 2, uma das vantagens do método é não ser influenciado pelos valores extremos.

No trabalho apresentado por Gomes et al (2010) para esse mesmo estudo, a alternativa indicada foi a aeronave LET 410 que, pelos métodos de Borda modificado e Borda original, é ordenada apenas na $4^{\mathrm{a}}$ colocação. Esse fato ocorre principalmente pela avaliação do critério conforto feito pelos gestores que consideraram apenas o espaço da cabine, não levando em consideração o espaço destinado ao bagageiro. Além disso, as diferenças entre o método de Borda modificado e o método NAIADE (Gomes et al, 2010) se justificam pois, ao usar a entropia, o método NAIADE torna-se altamente dependente das alternativas irrelevantes.

\section{Conclusão}

Em relação às alternativas utilizadas neste estudo, optou-se por aeronaves de capacidade máxima de dezenove passageiros, o que reduziu significativamente o universo de alternativas possíveis. A escolha por esse corte deu-se apenas por conta da estratégia assumida pela empresa, por isso, nenhum estudo mais aprofundado com alternativas que excedam esse nível de corte foi realizado. É possível que um estudo mais abrangente, no que diz respeito ao número de alternativas, aponte para uma maior viabilidade com aeronaves maiores.

Os critérios foram definidos de acordo com os valores de gestores de uma empresa préoperacional. Mesmo que tenham sido definidos com o maior rigor possível, é provável que o elenco de critérios importantes se modifique quando da operação efetiva. Uma válida sugestão para um estudo futuro é repetir esta análise depois de iniciadas as operações, para que se torne uma ferramenta útil quando da decisão pelo aumento de frota.

O método empregado foi fruto de uma escolha norteada por algumas características observadas sobretudo pelo analista de decisão. Em atendimento ao objetivo primário deste 
trabalho, a aplicação do método de Borda Modificado com utilização das Medianas definiu a alternativa que melhor refletia o que era buscado pela administração da empresa em análise.

Recomenda-se, portanto, ao decisor, a opção pela aeronave Beechcraft 1900, já que esta destacou-se como a que melhor atende as especificações e valores buscados no equipamento para o desempenho das atividades pretendidas.

O método de Borda Modificado com utilização das Medianas permitiu contornar as distorções do método de Borda original, já descritas ao longo deste trabalho; além de ser pouco dependente das posições extremas. É importante destacar que outros métodos podem ser utilizados para tentar resolver o mesmo tipo de problema. A comparação deste trabalho com outro que tente resolver o mesmo problema com outra metodologia é interessante, já que torna possível a validação do modelo matemático praticado neste trabalho.

\section{Referências}

Airliners.net (2009) Aircraft and aircraft carrier data. Disponível em www.airliners.net/aircraft-data.

Arrow, K. J. (1951) Social choice and individual values. New York: Wiley.

Arrow, K. J. (1950) A difficulty in the concept of social welfare. Journal of Political Economy, vol. 58, n. 4, pp. 328-346.

Bercovitz, J. e Mitchell, W. (2007) When is more better? The impact of business scale and scope on long-term business survival, while controlling for profitability. Strategic Management Journal, vol. 28, pp. 61-79.

Brandt, F. (2009) Some remarks on Dodgson's voting rules. Mathematical Logic Quartely, vol. 55, n. 4, pp. 460-463.

Brasil (1986) Lei No. 7.565, de 19 de Dezembro de 1986. Código Brasileiro de Aeronáutica (substitui o Código Brasileiro do Ar). Disponível em www.anac.gov.br.

Brasil (2003a) Agência Nacional de Aviação Civil. RBHA 119 - Requisitos operacionais: operações domésticas, de bandeira e suplementares. Regulamento Brasileiro de Homologação Aeronáutica. Disponível em www.anac.gov.br.

Brasil (2003b) Agência Nacional de Aviação Civil. RBHA 135 - Requisitos operacionais: operações complementares e por demanda. Regulamento Brasileiro de Homologação Aeronáutica. Disponível em www.anac.gov.br.

Brasil (2005) Lei No. 11.182, de 27 de Setembro de 2005. Cria a Agência Nacional de Aviação Civil ANAC, e dá outras providências. Disponível em www.anac.gov.br.

Chang, Y. H. e Yeh, C. H. (2002) A survey analysis of service quality for domestic airlines. European Journal of Operational Research, vol. 139, n. 1, pp. 166-177.

Chang, Y. H. e Yeh, C. H. (2001) Evaluating airline competitiveness using multiattribute decision making. Omega, vol. 29, n. 5, pp. 405-415. 
Clímaco, J. C. N. ( 2003) A critical reflection on optimal decision, European Journal of Operational Research, vol. 153, pp. 506-516.

Dias, L. M. C., Almeida, L. M. A. T. e Clímaco, J. C. N. (1996) Apoio multicritério à decisão. Coimbra: Faculdade de Economia da Universidade de Coimbra.

Dias, L. C. e Clímaco, J. C. N. (2000) Additive aggregation with variable interdependent parameters: The VIP analysis software. Journal of the Operational Research Society, vol. 51, n. 9, pp. 10701082.

Familytales.org (2009) Benjamin Franklin letter to Joseph Priestley, 19 September 1772. Disponível em www.familytales.org.

Gomes, L. F. A. M. (2007) Teoria da decisão. São Paulo: Pioneira Thomson Learning.

Gomes, L. F. A. M., Araya, M. C. G. e Carignano, C. (2004) Tomada de decisões em cenários complexos. São Paulo: Thomson.

Gomes, L. F. A. M., Soares de Mello, J. C. C. B. e Fernandes, J. E. M. (2010) Escolha de aeronave para empresa de voos charter: uma análise de decisão. Anais do IX Simpósio Brasileiro de Transporte Aéreo, SITRAER, Manaus, vol. 1, pp. 343-354.

Gomes Júnior, S. F. e Soares de Mello, J. C. C. B. (2007) Avaliação dos pilotos no campeonato mundial de Fórmula 1 no ano de 2006 utilizando modelo DEA com restrições cone rattio não arquimedianas. Anais do XXXIX Simpósio Brasileiro de Pesquisa Operacional, SBPO, Fortaleza, pp. 269-280.

International Civil Aviation Organization - ICAO (2004) Anex 14 to the Convention on International Civil Aviation: Aerodromes. Montreal.

Kangas, A., Laukkanen, S. e Kangas, J. (2006) Social choice theory and its applications in sustainable forest management-a review. Forest Policy and Economics, vol. 9, n. 1, pp. 77-92.

Kladroba, A. (2000) The problem of aggregation arising in the process of building rankings: dome temarks with the example of the Formula 1 Championship 1998 [Das aggregationsproblem bei der erstellung von rankings: einige anmerkungen am beispiel der Formel 1 Weltmeisterschaft 1998]. Jahrbucher fur Nationalokonomie und Statistik, vol. 220, n.3, pp. 302-314.

Laukkanen, S., Palander, T. e Kangas, J. (2004) Applying voting theory in participatory decision support for sustainable timber harvesting. Canadian Journal of Forest Research, vol. 34, n. 7, pp. 1511-1524.

Leskinen, P., Kangas, A. S. e Kangas, J. (2004) Rank-based modelling of preferences in multi-criteria decision making. European Journal of Operational Research, vol. 158, n. 3, pp. 721-733.

Levin, J. e Nalebuff, B. (1995) An introduction to vote-counting schemes. Journal of Economics Perspectives. vol. 9, n. 1, pp. 3-26.

Pinheiro, M. C. e Soares de Mello, J. C. C. B. (2005) Ordenação dos aeroportos do Brasil através do apoio de análises multicritério. Anais do XXXVII Simpósio Brasileiro de Pesquisa Operacional, SBPO, Gramado.

Pomerol, J. C. e Barba-Romero, S. (2000) Multicriterion decision in management: principles and practice. Kluwer Academic Publishers.

Rahman, J. M. B. e Gomes, L. F. A. M. (2008) Aplicação do método Electre I a um problema de transporte aéreo regional. Revista Pesquisa Naval, vol. 10, pp. 41-53.

Rocha, R. B. e Cavalcanti Netto, M. A. (2002) A data envelopment analysis model for rank ordering suppliers in the oil industry. Pesquisa Operacional, vol. 22, n. 2, pp. 123-132.

Roy, B. e Bouyssou, D. (1993) Aide multicritère à la décision: méthods et cas. Ed. Economica, Paris. 
Saaty, T. L. (2005) The analytic hierarchy and analytic network processes for the measurement of intangible criteria and for decision-making. In: Figueira, J., Greco, S. e Ehrgott, M. (editores) Multiple-criteria decision analysis. State of the art surveys. New York: Springer.

Silveira, J. Q., Pereira, E. R., Correia, T. C. V. D., Soares de Mello, J. C. C. B., Clímaco, J. C. N. e Angulo Meza, L. (2008) Avaliação da eficiência das companhias aéreas brasileiras com uma variação do modelo de Li e Reeves. Engevista, vol. 10, pp. 145-15.

Smith, W. D. (2006) Descriptions of single-winner voting system. Disponível em math.temple.edu/ wds/homepage/votedesc.pdf. Acesso em 10/01/2011.

Soares de Mello, J. C. C. B., Gomes, E. G., Gomes, L. F. A. M., Biondi Neto, L. e Leta, F. R. (2003) Seleção de rota aérea com o uso do apoio multicritério à decisão. Engevista, vol. 5, n. 10, pp. 71-84.

Soares de Mello, J. C. C. B., Gomes, E. G., Biondi Neto, L. e Angulo Meza, L. (2005) Avaliação do tamanho de aeroportos portugueses com relações multicritério de superação. Pesquisa Operacional, vol. 25, n. 3, pp. 313-330.

Soares de Mello, J. C. C. B., Gomes, L. F. A. M., Gomes, E. G. e Soares de Mello, M. H. C. (2005) Use of ordinal multi-criteria methods in the analysis of the Formula 1 world championship. Cadernos EBAPE.BR, vol. 3, n. 2, pp. 2-8.

Teodorović, D. (1985) Multicriteria ranking of air shuttle alternatives. Transportation Research Part $B$, vol. 19, n. 1, pp. 63-72.

Valladares, G. S., Gomes, E. G., Soares de Mello, J. C. C. B., Pereira, M. G., Anjos, L. H. C., Ebeling, A. G. e Benites, V. M. (2008) Análise dos componentes principais e métodos multicritério ordinais no estudo de organossolos e solos afins. Revista Brasileira de Ciência do Solo, vol. 31, n. 1, pp. 285-296.

Wang, T. C. e Chang, T. H. (2007) Application of TOPSIS in evaluating initial training aircraft under a fuzzy environment. Expert Systems with Applications, vol. 33, n. 4, pp. 870-880.

Wang, Y. J. (2008) Applying FMCDM to evaluate financial performance of domestic airlines in Taiwan. Expert Systems with Applications, vol. 34, n. 3, pp. 837-1845.

Yoo, K. E. e Choi, Y. C. (2006) Analytic hierarchy process approach for identifying relative importance of factors to improve passenger security checks at airports. Journal of Air Transport Management, vol. 12, n. 3, pp. 135-142.

Zadeh, L. A. (1975) The concept of a linguistic variable and its application to approximate reasoning. Information Sciences, vol. 1, pp. 119-249. 